\title{
Lemniscal and extralemniscal compartments in the VPM of the rat
}

\author{
Sebastian Haidarliu*, Chunxiu Yu, Naama Rubin and Ehud Ahissar \\ Department of Neurobiology, The Weizmann Institute of Science, Rehovot, Israel \\ Edited by: Kathleen S. Rockland, RIKEN Brain Science Institute, Japan \\ Reviewed by: Vivien A. Casagrande, Vanderbilt University, USA \\ Noritaka Ichinohe, RIKEN Brain Science Institute, Japan
}

The ventral posteromedial thalamic nucleus (VPM) of the rat contains at least two major vibrissa-representing compartments: the dorsomedial (VPMdm), which belongs to the lemniscal afferent pathway, and the ventrolateral (VPMvl), which belongs to the extralemniscal afferent pathway. Although input-output projections and functional characteristics that distinguish these two compartments were recently clarified, a comprehensive structural analysis of these compartments and the border between them was lacking. This paper addresses structural and functional relationships between the VPMdm and VPMvl. We found that the size of the VPM is almost constant across individual rats. Next, we computed a canonical map of the VPM in the oblique plane, where structural borders are best visualized. Using the canonical map, and sequential slices cut in oblique and coronal planes, we determined the border between the VPMdm and VPMvl in the standard coronal plane, and verified it with in vivo extracellular recordings. The position of the border between these two vibrissal sub-nuclei changes along the rostrocaudal extent within the VPM due to the relative sizes of these sub-nuclei at any point. The border between the VPMdm and VPMvl, which was revealed by this technique, can now be included in atlases of the rat brain and should facilitate experimental correlation of tactile functions with thalamic regions.

Keywords: brain mapping, vibrissal representation, parallel pathways, cytochrome oxidase, active touch

\section{INTRODUCTION}

Since the original description of spatial organization of somesthetic regions within the rat thalamus (Davidson, 1965; Emmers, 1965), known as the ventrobasal complex (VB), the structure and function of thalamic relay stations have been described in detail (Groenewegen and Witter, 2004; Macchi, 1983; Price, 1995). In rat brain atlases (Paxinos and Watson, 1998; Swanson, 1992) and reviews of thalamic structure and function (Groenewegen and Witter, 2004; Price, 1995), the ventral posteromedial thalamic nucleus (VPM) is considered to be, and is mapped as, a separate thalamic anatomical structure that includes the gustatory sub-nucleus. Functionally, the VPM was classified as a subcortical relay station of the lemniscal pathway (Sugitani et al., 1990; Vahle-Hinz and Gottschaldt, 1983; Waite, 1973) that also contains periodontal mechanoreceptive (Tabata et al., 2002), as well as taste, temperature, and tactile oral sensory representations (Cechetto and Saper, 1987; Emmers, 1965; Verhagen et al., 2003). Thus, the VPM was considered as an obligatory relay station in the sensory pathways mentioned above (Kosar et al., 1986; Price, 1995; Saper, 1995).

*Correspondence: Sebastian Haidarliu, Department of Neurobiology, The Weizmann Institute of Science, 76100 Rehovot, Israel. e-mail: sebastian.haidarliu@weizmann.ac.il Received: 11 July 2008; paper pending published: 04 August 2008; accepted: 02 September 2008; published online: 12 September 2008.

Citation: Front. Neuroanat. (2008) 2: 4. doi: 10.3389/neuro.05.004.2008

Copyright $\odot 2008$ Haidarliu, Yu, Rubin and Ahissar. This is an open-access article subject to an exclusive license agreement between the authors and the Frontiers Research Foundation, which permits unrestricted use, distribution, and reproduction in any medium, provided the original authors and source are credited.
Recently, the vibrissal compartment of the VPM was shown to be supplied by two different ascending pathways [from the principal trigeminal nucleus (PrV) to the dorsomedial part of the VPM (VPMdm), and from the caudal division of the interpolaris nucleus $(\mathrm{SpVi})$ to the ventrolateral part of the VPM (VPMvl)] and to project to different targets (Pierret et al., 2000; Veinante and Deschenes, 1999). Functionally, the two sub-nuclei were found to convey different sensory signals: contact signals by the VPMvl and complex whisking-touch signals by the VPMdm (Yu et al., 2006). Yet, the structural boundaries between the two sub-nuclei had only been partially described. A third pathway, ascending through the "heads" of the barreloids, has recently been reported (Urbain and Deschenes, 2007).

The VPM contains head representations (Waite, 1973), with its dorsolateral part containing representations of the contralateral vibrissae (Sugitani et al., 1990; Vahle-Hinz and Gottschaldt, 1983). In the VPM, each vibrissa is represented by a cluster of neurons called a "barreloid". These arrays of rod-shaped domains were first discovered in the medial part of the mouse VB by Van der Loos (1976). In the rat VPM, patches of high activity of succinic dehydrogenase (a mitochondrial enzyme) and a segmentation of retrogradely labeled thalamic neurons are already present during the early postnatal period (Belford and Killackey, 1979; Ivy and Killackey, 1982). Barreloids are also observed in adult rats (Land and Akhtar, 1987; Land and Simons, 1985). The spatial orientation of the long axes of barreloids appeared to be different from the three traditional orthogonal cutting planes of the brain (coronal, horizontal and sagittal). Thus, for clarification of the three-dimensional arrangement of barreloids in the VPM, special cutting planes were used (Haidarliu and Ahissar, 
2001; Land et al., 1995). In the rat, we found that barreloids are rod-shaped, with their long axes directed from dorsomedial to ventrolateral, and a length gradient that increases diagonally from barreloid A1 to barreloid E4 (Haidarliu and Ahissar, 2001). Individual barreloids are most easily seen in young animals, but are also distinguishable in adults.

In previously proposed three-dimensional models of barreloid arrangement in the VPM (Haidarliu and Ahissar, 2001; Land et al., 1995), barreloids reconstructed from cytochrome oxidase (CO)-stained sections do not occupy all the area assigned to the VPM in current rat brain atlases. Anterograde tracing with biotinylated dextran amine injected into the SpVi, and retrograde tracing with Fluoro-Gold injected into a single cortical barrel column, facilitated a three-dimensional reconstruction in which barreloids consisted of a core located in the VPMdm, and a tail that extended ventrolateral and caudally into the VPMvl (Pierret et al., 2000). In Nissl-stained slices, these subdivisions cannot be distinguished in the rat VPM. Staining for CO activity revealed homologous regions in the VPM of rodents, but the border between the VPMdm and VPMvl could not be visualized in traditional section planes (Land and Simons, 1985; Pierret et al., 2000). Although the transition from the core to tail regions of barreloids was assumed to occur at the VPMdm/ VPMvl border (Pierret et al., 2000), the exact position of the border and its extent was unclear. Recently, it was concluded that no cytoarchitectonic feature or immunohistochemical stain delineated the boundary of the VPMvl within the VPM (Bokor et al., 2008).

Previously, we showed that (1) the border between the two VPM compartments (VPMdm and VPMvl) can be clearly identified in both young and adult rats by cutting brains at specific angles and staining for CO activity (Haidarliu and Ahissar, 2001), and (2) the neuronal populations of the two VPM compartments exhibit different electrophysiological characteristics during whisking and object touch: VPMvl neurons convey contact-time information whereas VPMdm neurons convey complex information related to both whisking and touch (Yu et al., 2006). We also described a method of normalization of anatomical VPM coordinates that enables translation of individual parameters of recording sites (lesions), visualized by CO-staining of coronal slices, into an oblique image, with the VPMdm clearly separated from the VPMvl (Yu et al., 2006). This procedure permits translation of lesions observed in thalamic coronal slices into an oblique canonical section of the VPM that facilitates viewing of recording sites in relevant compartments of the VB complex. Here, we modified this method to project the anatomical border between the VPMdm and VPMvl, which can be identified in consecutive oblique slices through the thalamus, onto consecutive coronal slices. Determination of segmentation of the VPM in the coronal plane should allow division of the vibrissal part of the VPM into two sub-nuclei, and inclusion of the border between the VPMdm and VPMvl in rodent brain atlases.

\section{MATERIALS AND METHODS}

\section{ANIMALS}

Histological preparations of the brains of eight 6-day-old, three 9-day-old, and 28 adult Albino Wistar rats, weighing 11-13, 21-23, and 200-300 g, respectively, were subjected to morphological analysis, including mapping of complete sets of slices through the VPM in coronal and two non-traditional cutting planes. Morphological and physiological data from histological preparations, and neuronal recordings of another 40 adult rats (200-300 g) with thalamic lesions (Yu et al., 2006), were also used. All animals were obtained from The Animal Breeding Unit of this Institute. Procedures for animal maintenance, manipulations, and surgeries were approved by the Institute's Animal Care and Use Committee, and conform to the "Principles of Laboratory Animal Care” (NIH publication No. 86-23, revised 1985).

\section{RECORDINGS}

Recordings were conducted as previously described (Szwed et al., 2003). Briefly, surgery was performed under general anesthesia (urethane; $1.5 \mathrm{~g} / \mathrm{kg}$, i.p.), with supplemental doses of anesthetic (10\%) being administered when required. Atropine methyl nitrate $(0.3 \mathrm{mg} / \mathrm{kg}$, i.m. $)$ was administered to prevent respiratory complications. Anesthetized animals were secured in a stereotaxic device (SR-6; Narishige, Japan), and their body temperature maintained at $37^{\circ} \mathrm{C}$. An opening was made in the skull overlying the right thalamus, and tungsten microelectrodes (0.5-1 M $\Omega$; Alpha Omega Engineering; Nazareth, Israel) were lowered, according to known stereotaxic coordinates of the POm and VPM, until units drivable by whisker stimulations were encountered. Up to four electrodes, spaced $0.33 \mathrm{~mm}$ from each other, were lowered in parallel in each recording session. Standard methods for singleunit recordings were used. Single-units were sorted by spike templates. Units were considered single only if they had homogenous spike shapes that did not overlap with other units or noise, and if they exhibited refractory periods of $>1 \mathrm{~ms}$ in their autocorrelation histograms. Artificial whisking was induced as described in Szwed et al. (2003). Briefly, the buccal branch of the facial nerve was cut, and its distal end mounted on a pair of silver electrodes. Bipolar, rectangular electrical pulses $(0.5-4.0 \mathrm{~V}, 40 \mu$ s duration) were applied through an isolated pulse stimulator (Model 2100; A-M Systems, Sequim, WA, USA) at $83 \mathrm{~Hz}$, the lowest frequency that still produces continuous whisker movement. Whisker movements were recorded at 1000 frames/s with a fast digital video camera (MotionScope PCI 1000; Redlake, San Diego, CA, USA). Artifacts produced by electrical stimulation were isolated by an online spike-sorter (MSD-3.21; Alpha-Omega Engineering) and removed from unit recordings.

\section{HISTOLOGY}

Rats were euthanized with Pental (0.1-0.5 ml pro capito, i.p.), perfused transcardially with $2.5 \%$ glutaraldehyde, $0.5 \%$ paraformaldehyde, and 5\% sucrose in $0.1 \mathrm{M}$ phosphate buffer, $\mathrm{pH}$ 7.4, and then decapitated. Their brains were excised and stored in postfixation solution until sectioned, usually within $36 \mathrm{~h}$. Tissue slices were prepared with a sliding microtome (SM 2000R; Leica Instruments, Germany) supplemented with a freezing unit (K400; Microm International, Germany). Histological procedures were identical to those previously employed (Haidarliu and Ahissar, 2001). Briefly, brains were sliced into $60 \mu \mathrm{m}$ thick slices, in a traditional coronal plane and in two planes that reveal barreloid arcs or entire barreloid fields. We found that the border between the two VPM compartments is most clearly seen in oblique sections, at $50^{\circ}$ to the horizontal plane, from dorsomedial to ventrolateral. All slices were stained for CO activity as previously described (Haidarliu and Ahissar, 2001), coverslipped with Entellan, and subjected to light microscopy (Zeiss, Oberkochen, Germany). In a few cases, sections stained for CO activity were also stained with cresyl violet (Haidarliu et al., 1999) to visualize cell bodies. Brightfield images were captured by Adobe Premiere 5.0 software (Adobe Systems, Mountain View, CA, USA), and imported into 
Adobe Photoshop 4.0. During editing of the resulting images, only minimal adjustments in contrast and brightness were made.

\section{PROCESSING OF CORONAL SLICES}

In coronal slices of the thalamus, the most rostral barreloid row of the VPM (row E) appeared approximately $-2.3 \mathrm{~mm}$ caudal to bregma, according to the atlas of Paxinos and Watson (1998). The most caudal row (row A) disappeared about $-4.3 \mathrm{~mm}$ from bregma. In the rostrocaudal direction, the VPM was represented by $22-26$ consecutive $60 \mu \mathrm{m}$ thick sections when cut after postfixation with 15-20\% sucrose. In each section, the position of the "tip", i.e., the dorsolateral pole of the VPM, was determined, and its distance from the midline and bregma measured. For alignment of coronal sections, the distance from the VPM tip to bregma and to midline appeared sufficient.

\section{PREPARATION OF OBLIQUE SLICES AND OF AN OBLIQUE CANONICAL MAP OF THE VPM}

After overnight postfixation, entire rat brains were cut into two hemispheres, and left in the postfixation solution for an additional $24 \mathrm{~h}$. The dorsolateral and ventromedial poles of each hemisphere were then cutoff at an angle of $50^{\circ}$ to the horizontal plane, as previously indicated (Haidarliu and Ahissar, 2001). When cut correctly, the two parts of the hippocampus (dorsomedial and ventrolateral) were visible in the sectioning plane, and were separated by a field that stained intensively for $\mathrm{CO}$ and contained the corpora geniculata. Usually, the VPM started in slices $1 \mathrm{~mm}$ deeper than the beginning of the corpora geniculata, and continued in oblique slices for another 2.6-3.0 mm. In oblique sections, clear compartmentalization of the vibrissal part of the VPM was observed for about half of the VPM, starting from its dorsolateral pole, i.e., from the straddlers in the part represented by macrovibrissae, and was preserved until the parvicellular part of the ventral posterolateral thalamic nucleus (VPLpc) appeared in the slices adjacent to the most ventrocaudal sector of the VPM. In the rat brain atlas of Paxinos and Watson (1998), the VPM contour in the horizontal plane can be characterized by a simple curve. We found that the clearest and most representative images of the vibrissal part of the VPM correspond to the arcs 3-5 of the barreloid field (Figure 1A). In such oblique slices, the contours of the entire VPM, and of individual barreloids were manually outlined. The contours of the VPM thus obtained were superimposed, rotated, and aligned, and an average contour was computed for this set of curves (Figure 1B, red lines). It can be seen that VPM has a simple outline, and its shape can be considered as an asymmetric ellipse, with the major axis $(j)$ directed rostrocaudal, and the minor axis $(\mathrm{n})$, directed from dorsomedial to ventrolateral (Figure 1C).

This averaged VPM contour, with the border outlined between the VPMdm and VPMvl (Figure 1C, solid black lines), was used as a canonical image that represents a valid portrayal of the VPM compartments for translation of functional sites from oblique to coronal sections. The correspondence of the canonical image to the real relationships between VPM compartments in the oblique plane was studied by its superimposition on consecutive oblique sections of the VPM. The canonical image satisfactory fits both the VPMdm and VPMvl compartments in the oblique slices, starting from arc 3 until the appearance of most of the thin barreloids. Thus, structural relationships between the VPMdm and VPMvl compartments, which were revealed and mapped in the canonical map, are maintained in oblique sections of the VPM for $\sim 1 \mathrm{~mm}$ along its oblique axis.

\section{TRANSLATION OF THE BORDER BETWEEN THE VPMdm AND VPMvI FROM THE OBLIQUE TO THE CORONAL PLANE}

Previously, we proposed a method for translation lesion coordinates from the coronal to oblique plane (Yu et al., 2006). Here, we propose a method of coordinate translation, from the oblique to coronal plane, that consists of consecutive determinations of the position of borderline fragments that correspond to each of the consecutive oblique slices of the dorsolateral part of the VPM. The vertical solid line $\mathrm{b}_{1}$ (Figure 2A) corresponds to the 13th coronal slice of the VPM (Figure 2B). For clarity, a magnified contour of the VPM from the coronal slice 13 (Figure 2B) is depicted in Figure 2C, and the position of the oblique slice relevant to canonical image (Figure 2A) is marked by the solid line $\mathrm{b}_{2}$. For coordinate translation, the border between the VPMdm and VPMvl in the 13th coronal slice at the level of arc $3\left(\mathrm{x}_{1}\right.$ in Figure 2C) must be located. The position of each of 24 consecutive coronal slices along the length of the rostrocaudal extent of the VPM is indicated by the $x$-axis in Figure 2A. The VPMdm extent $\left(a_{1}\right)$ is measured as the distance from the VPM/POm border to the VPMdm/VPMvl border; $b_{1}$ is the diameter of the VPM in this oblique slice. The length of the core of the barreloids in coronal slice 13 is derived by $a_{2}=a_{1} \times b_{2} / b_{1}$.
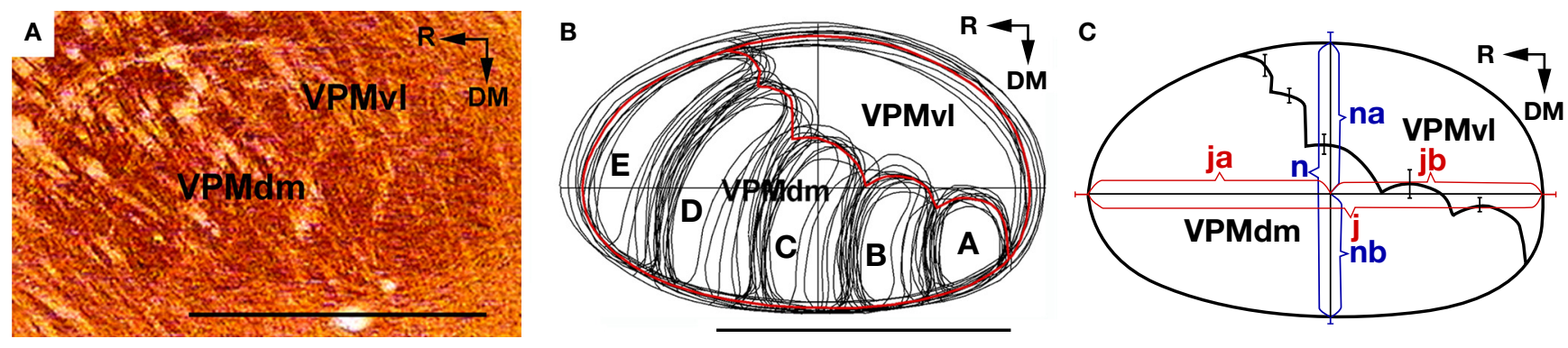

Figure 1 | Constructing a canonical map of the VPM and of the border between VPMdm and VPMvl in an oblique plane. (A) Digital photomicrograph of an oblique $\left(50^{\circ}\right.$ to the horizontal plane, from dorsomedial to ventrolateral) slice through the VPM stained for $\mathrm{CO}$ activity. (B) Manually outlined contours of the VPM and of barreloids in oblique slices from 11 adult rats. The barreloids A, B, C, D, and E are arranged in an arc. Solid red lines, averaged contours of the VPM and of the border between the VPMdm and VPMvl compartments. (C) Canonical image of the VPM in the oblique plane (solid black lines). j, major axis, corresponding to the maximum diameter; ja and jb, major semi-axes; $n$, minor axis; na and nb, minor semi-axes. SD for each semi-axis is represented by a bar. Bars on the $\mathrm{dm} / \mathrm{vl}$ borderline show the SDs for individual barreloids in the arc. DM, dorsomedial; R, rostral; VPMdm, dorsomedial compartment of the ventral posteromedial thalamic nucleus; VPMvl, ventrolateral compartment of the VPM. Scale bars are $1 \mathrm{~mm}$. 
A

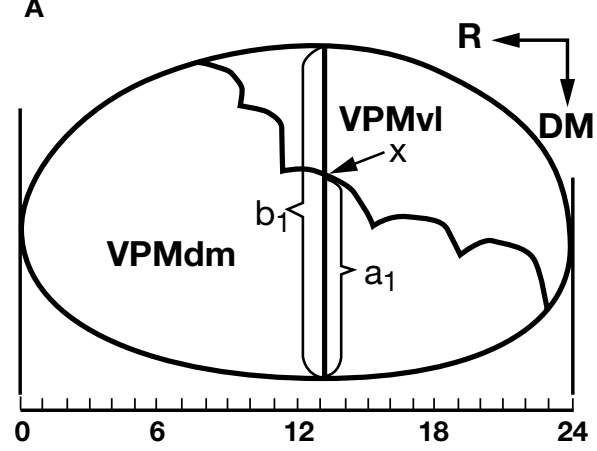

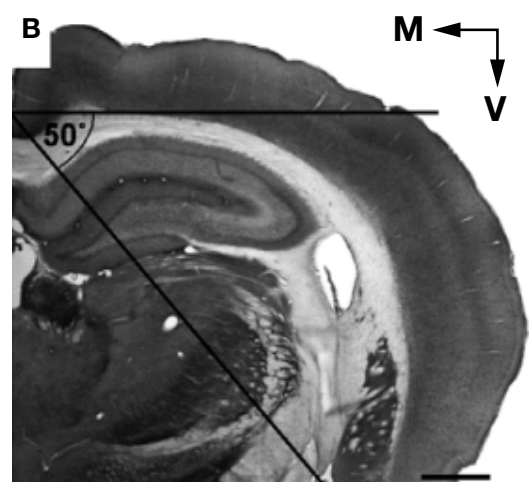

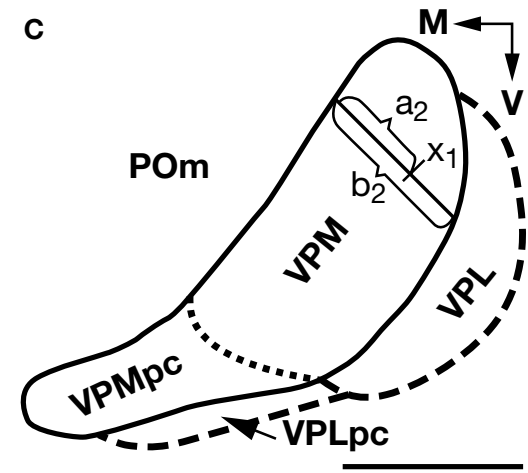

Figure 2 | Coordinate translation from the oblique to coronal plane. (A) Canonical contours of the VPM and of the border between VPMdm and VPMvl; $\mathrm{a}_{1}$ and $b_{1}$, mediolateral diameter of the VPMdm ("core") and the entire VPM, respectively; $x$, the border between VPMdm and VPMvl at the level of the coronal slice 13. (B) Digital photomicrograph of a 60- $\mu \mathrm{m}$ thick coronal slice (13th of 24 starting from the rostral border of the VPM). The horizontal plane and oblique $\left(50^{\circ}\right.$ to the horizontal plane) cutting plane are indicated by solid lines. (C) Contour of the VPM in the coronal slice in $(\boldsymbol{B}) \mathrm{a}_{2}$ and $\mathrm{b}_{2}$, diameter of the VPMdm and entire VPM, respectively; $x_{1}$, the border between VPMdm and VPMvl that corresponds to the position of the oblique canonical image shown in $\left.(\boldsymbol{A})\right]$. A dotted line separates the VPMpc from the rest of the VPM. DM, dorsomedial; M, medial; R, rostral; V, ventral; VPL, ventral posterolateral thalamic nucleus. Scale bars are $1 \mathrm{~mm}$.

The entire length of the border between the VPMdm and VPMvl in coronal slice 13 could be determined by performing this procedure on all, or a subset of, consecutive oblique slices of the vibrissal subdivision of the VPM. In each oblique slice, the distances from the VPM/POm border to the ventrolateral end of the barreloid core (denoted as $\mathrm{a}_{1}$ ), and to the VPM/VPL border (denoted $a b_{1}$ ), were measured, and the value for the distance $a_{1}$ was marked on a relevant image of the VPM in the coronal plane. Since segregation of the vibrissal part of the VPM into the VPMdm and VPMvl can be clearly seen after oblique sectioning of the rat brain at an angle of $\sim 50^{\circ}$ to the horizontal plane, from dorsomedial to ventrolateral, and histochemical staining for CO activity (Haidarliu and Ahissar, 2001; Yu et al., 2006), these two VPM compartments can be mapped for all oblique consecutive images through the vibrissal part of the VPM. In the rostrocaudal direction, the oval, and almost symmetric, shape of the VPM, with two clearly separated compartments in oblique slices, is conducive for translation of the position of the two compartments to the coronal plane.

\section{RESULTS \\ APPEARANCE OF VPM COMPARTMENTS IN AN OBLIQUE CUTTING PLANE}

In oblique rat brain slices, cut dorsomedial to ventrolateral at an angle of $50^{\circ}$ to the horizontal plane, staining for $\mathrm{CO}$ activity visualized barreloids arranged in arcs. In such slices, the VPM is easily recognized, with the dorsolateral end of the VPM becoming visible as a structure strongly stained for CO activity, medial to the reticular nucleus (Rt) and VPL. A series of slices that includes 6 (nonconsecutive) of 34 consecutive oblique slices through the right VPM (ventral view) of an adult rat is shown in Figure 3A. In slice 1, representations of four straddlers, together with more laterocaudally located supraorbital (SO) vibrissa representations are seen. The barreloids that represent straddlers have similar orientations, with their short axes directed rostrocaudal and their long axes directed ventrolateral. In adult rats, the rostrocaudal diameter of the cores of straddler representing barreloids varied from 180 to $220 \mu \mathrm{m}$, and the mediolateral length varied from 400 to $500 \mu \mathrm{m}$ (data not shown). The 4 th arc of vibrissal barreloids is represented in slice 6. Slices 2-6 contained representations of the first four arcs of mystacial vibrissae, with five barreloids present in each arc. Row E in slices 2-6 is composed of the most curved and longest barreloids, which form the rostral, and part of the lateral, border of the VPM, and which determine the convexity of the rostral contour of the VPM. In this mystacial vibrissa representing part of the VPM, the outlines of individual barreloids arranged in arcs (Figure 3B) can be determined, and the size gradient of barreloids, which were previously described (Haidarliu and Ahissar, 2001) is clearly seen. Slice 16 contains thin barreloids. These barreloids represent small hairs and hairy buccal pads rostral and ventral to mystacial vibrissae, respectively.

The cores of large barreloids (which represent macrovibrissae) and of thin barreloids (which represent microvibrissae and other face regions) occupy the dorsomedial part of the VPM. The laterocaudal part of the VPM contains the "tails" of the barreloids. Entire tails are not seen in individual oblique sections cut at $50^{\circ}$ to the horizontal plane, because of their curvilinear configuration, as shown by Pierret et al. (2000). In fact, only short fragments of tails that belong to barreloids seen in preceding slices are represented in these oblique slices. The VPMvl appears as a narrow strip at the level of the 1st barreloid arc in the first few slices, and typically becomes wider in the next 14-16 (60 $\mu \mathrm{m}$ thick) slices, with a length up to $\sim 1.5 \mathrm{~mm}$ along the ventrolateral border of the VPM.

At the level of the VPM represented by slice 22, thin barreloids are seen, but only in the rostral part of the VPM, and do not seem to possess tails. Instead, the ventrolateral parts of structures extensively stained for CO activity are present in the VPLpc, which separates the VPM from the medial lemniscus (ml). Further ventromedially (e.g., slice 28), oblique slices contain the VPMpc and VPLpc, which stain homogenously for CO activity. The final slices (see slice 34) contain only the VPMpc, which stains more extensively for CO activity than surrounding tissues. Contours of the VPM in relation to oblique slicing (Figure $3 \mathrm{~A}$ ) are depicted in Figure 3B. Three vertical lines cross these contours at -2.56 , -3.60 , and $-4.20 \mathrm{~mm}$ caudal to bregma, and correspond to the coronal positions of contours shown in Figure 3C. At $-2.56 \mathrm{~mm}$ caudal to bregma, coronal slice 6 contains only core components 
A

6
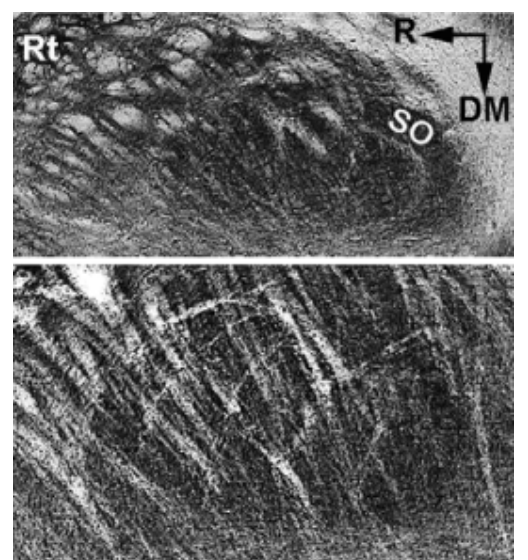

16

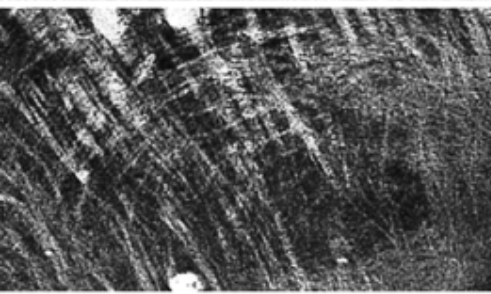

22

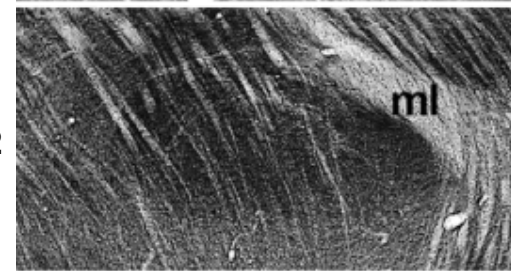

28

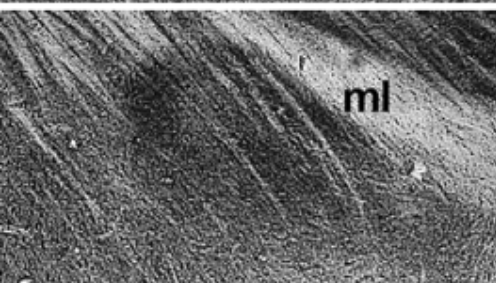

34

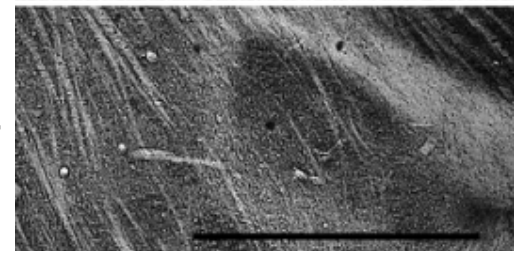

C

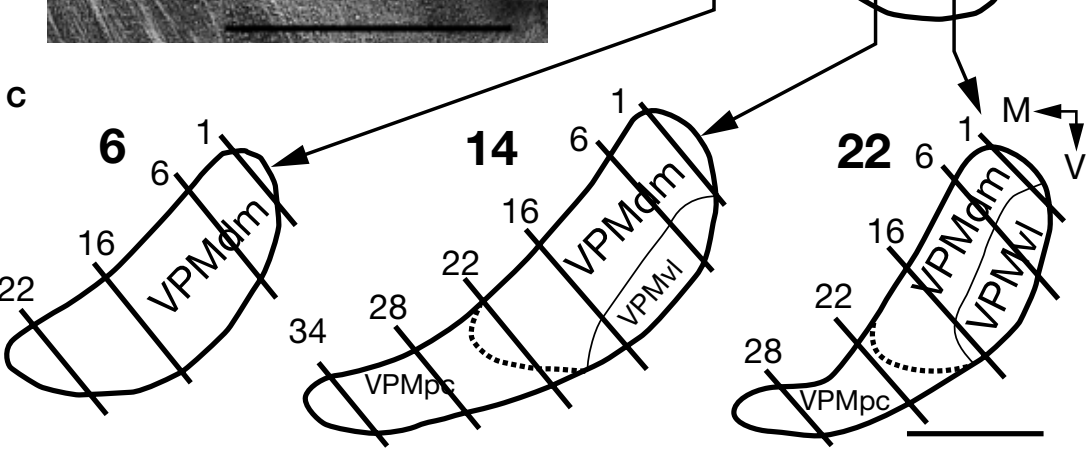

B

$-2.56-3.60-4.20$

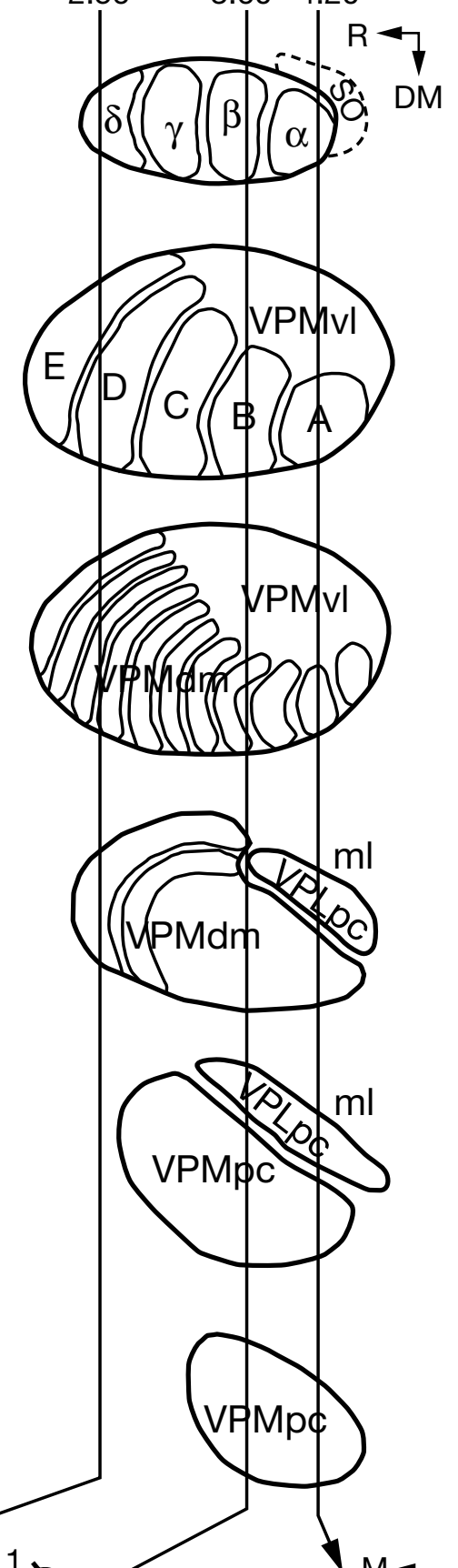



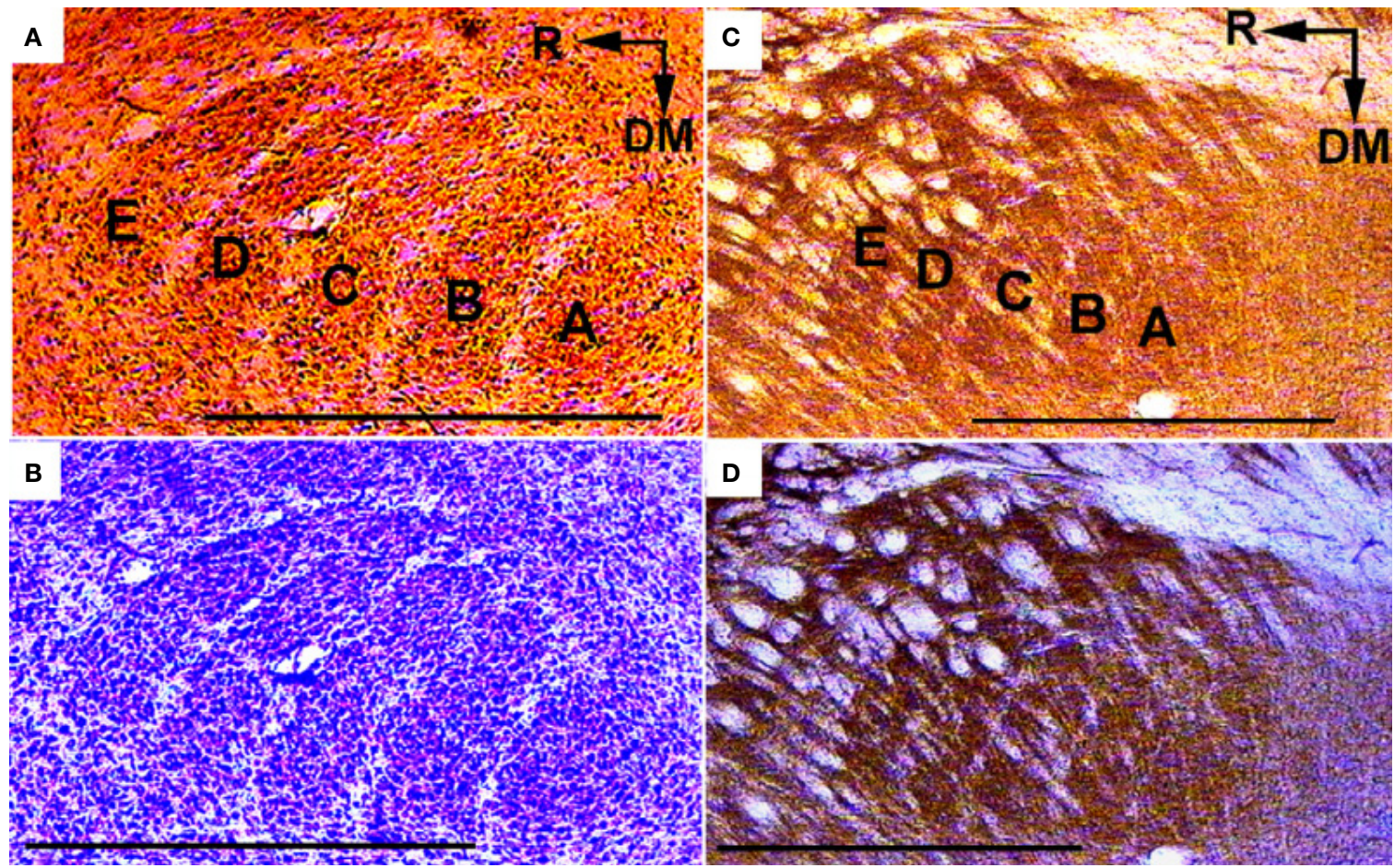

Figure 4 | Visualization of septa between barreloids and of the border between the VPMdm and VPMvl, in oblique slices of the thalamus. Preparations from young (6-day-old) $(\boldsymbol{A}, \boldsymbol{B})$ and adult $(\boldsymbol{C}, \boldsymbol{D})$ rats after staining for $\mathrm{CO}$ activity $(\boldsymbol{A}, \boldsymbol{C})$ followed by cresyl violet staining $(\boldsymbol{B}, \boldsymbol{D})$ of the same thalamic slices. $A, B, C$, $D$, and $E$, barreloids arranged in arcs; DM, dorsomedial; R, rostral. Scale bars are $0.5 \mathrm{~mm}(\boldsymbol{A}, \boldsymbol{B})$, and $1 \mathrm{~mm}(\boldsymbol{C}, \boldsymbol{D})$.

of the VPM. At $-3.60 \mathrm{~mm}$ caudal to bregma, coronal slice 14 also contains cores of barreloids, but its ventrolateral part is occupied by the tail compartment, the VPMvl. At $-4.20 \mathrm{~mm}$ from bregma, more than half of the mediolateral length of the VPM in coronal slice 22 is occupied by the VPMvl.

In oblique slices of young rat brains that were stained for $\mathrm{CO}$ activity (Figure 4A), and then stained with cresyl violet (Figure 4B), the border between the VPMdm and VPMvl, as well as the septa between barreloids in arcs, are clearly seen as areas poorly stained for $\mathrm{CO}$ and with low density of cell bodies. In corresponding and similarly stained oblique sections of adult rats, the cores of barreloids, as well as the border between the VPMdm and VPMvl, and the septa between the barreloids, are not as clear (Figures 4C,D). In the VPMvl, tails of barreloids can appear as small patches stained for CO activity, but with no regular pattern.

\section{APPEARANCE OF THE VPMdm AND VPMvI IN CORONAL SLICES}

In consecutively-cut coronal slices stained for $\mathrm{CO}$ activity, the VPM is distinguishable along its whole rostrocaudal length (see Figure 5A). For viewing progressive relationships between the VPMdm and VPMvl in the rostrocaudal direction, positions of coronal cuttings through the VPM (according to delineations by Paxinos and Watson, 1998) are shown in an oblique canonical image of the VPM (Figure 5C), along with corresponding coronal slices (Figure 5A), and enlarged contours of both the VPMdm and VPMvl (Figure 5B). In the most rostral slices, the VPM first appeared as an oval dark-brown spot about $-2.3 \mathrm{~mm}$ caudal to bregma (slice 1 in Figure 5A), and was surrounded by a less intensively stained VPL in the ventrolateral direction, and by the ventrolateral thalamic nucleus in the dorsomedial direction. In more caudal slices, the size of the VPM rapidly increased, becoming maximal between -3.3 and $-3.6 \mathrm{~mm}$ caudal to bregma (slices 12-15 in Figure 5A). Note that the consecutive rostral slices (slices 1-6) contain only the cores of barreloids (VPMdm). The tails, i.e., VPMvl, first appeared as a narrow strip at the border between the VPM and VPL (see slice 10), which continued to increase in more caudal slices (Figure 5B).

\section{MORPHOLOGICAL AND FUNCTIONAL CHARACTERISTICS OF THE VPMdm AND VPMvl}

In the rostral part of the VPM (slices 1-6 of the VPM in Figure 5B), the mediolateral extent of the VPM belongs only to the VPMdm. However, in the caudal part of the VPM, more than half of the mediolateral extent of the VPM belongs to the VPMvl. Since cytoarchitectonic subdivisions of the VPM cannot be observed in Nissl-stained coronal slices or slices stained for CO activity alone (Haidarliu and Ahissar, 2001; Land and Simons, 1985; Pierret et al., 2000), it cannot be determined to which compartment ( $\mathrm{dm}$ or $\mathrm{vl}$ ) a lesion in the ventrolateral half of the VPM belongs (see slices 9 and 16 in Figures 6A,B, respectively). In these examples, each slice contains a lesion indicated by an arrow, and both lesions are closer to the border with the VPL than the border with the POm. The locations of lesions in these slices were translated to canonical images in the oblique cutting plane (Figures 6C,D). The lesion in Figure 6A appeared to be located in the core of a barreloid in row D. In fact, the principal whisker identified for this recording site was D3. The location of the lesion in Figure 6B corresponded to the VPMvl, and the neurons recorded by that electrode responded to stimulation of two whiskers (C1 and $\mathrm{C} 2)$. The contours of the VPM, and the lesion locations for Figures $6 \mathrm{~A}, \mathrm{~B}$ are depicted in Figures 6E,F, respectively. Since the VPMvl compartment of Figure $6 \mathrm{~F}$ extends more medially than that of Figure $6 \mathrm{E}$, the 
A

C $\quad \begin{array}{lllllllll}0 & 3 & 6 & 10 & 12 & 15 & 18 & 21 & 23\end{array}$

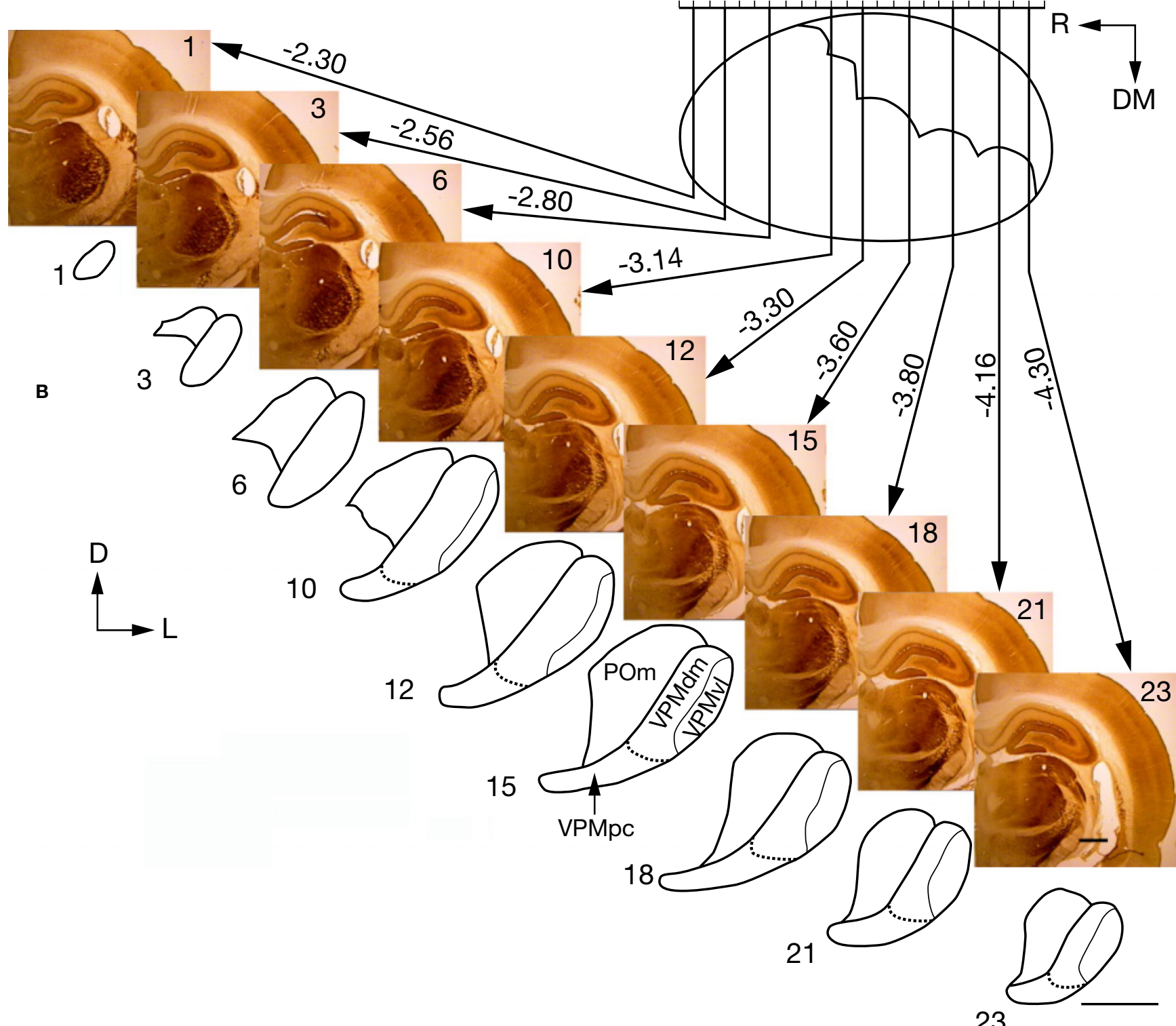

23

Figure 5 | Maps of the VPMdm and VPMvl in the coronal plane of an adult rat, at various distances from the bregma. (A) Coronal slices through the VPM, ordered from rostral to caudal. Numbers indicate the selected consecutive coronal slices. (B) Outlines of the VPMdm and VPMvl for coronal slices shown in (A). (C) Canonical map of the VPM cut mediolateral at $50^{\circ}$ to the horizontal plane. Numbers of the depicted consecutive slices are at the top. Distances from the bregma (according to Paxinos and Watson, 1998) of the cutting positions for the depicted coronal slices are shown. D, dorsal; DM, dorsomedial; L, lateral; P0m, posteromedial complex; R, rostral. Scale bars are $1 \mathrm{~mm}$.

lesion in it was localized in the VPMvl compartment, whereas that of Figure 6E, although a similar distance from the POm border, was localized in the VPMdm. The activities of the neurons recorded from these two VPM sites during artificial whisking (Szwed et al., 2003) are shown in Figures 6G,H, respectively. When extracellular recordings were performed from these sites during whisking in air and during object touch, neuron(s) from the site depicted in Figure 6A displayed a Whisking/Touch response pattern $(\mathrm{W}+\mathrm{T})$, characteristic of VPMdm neurons, while neuron(s) recorded from the site depicted in Figure 6B displayed a Touch response pattern (T), characteristic of VPMvl neurons (Yu et al., 2006).

Our results demonstrate that whether or not an electrode tip is in the VPMdm or VPMvl cannot be determined by the distance of a recording site from the border between POm and VPM. For example, the recording sites in Figure $6 \mathrm{~A}$ (slice 9 from rostral) and Figure 6B (slice 16) are approximately the same distance from the POm/VPM border, but the characteristics of their responses were typical of two different VPM compartments: the VPMdm and VPMvl, respectively (Figures 6G,H).

\section{DISCUSSION}

We describe, for the first time, the border between the VPMdm and VPMvl in consecutive coronal slices of rat brains. This border can now be included in standard rat brain atlases, and will facilitate analysis of physiological and anatomical data according to functional subdivisions of the rat somatosensory 


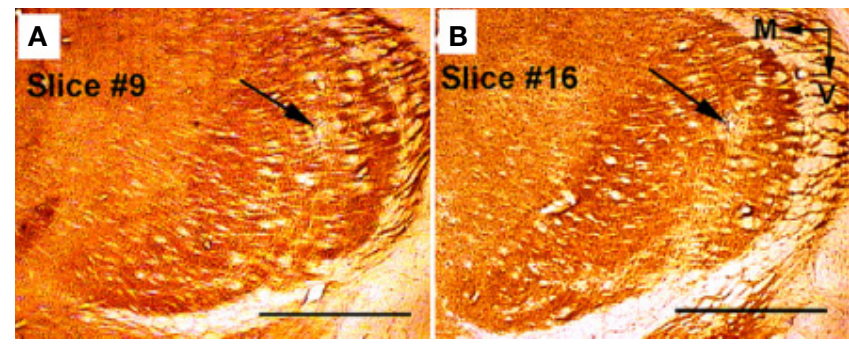

C

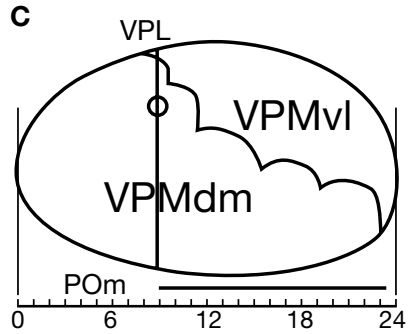

D

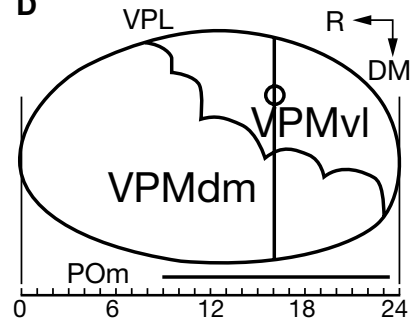

E
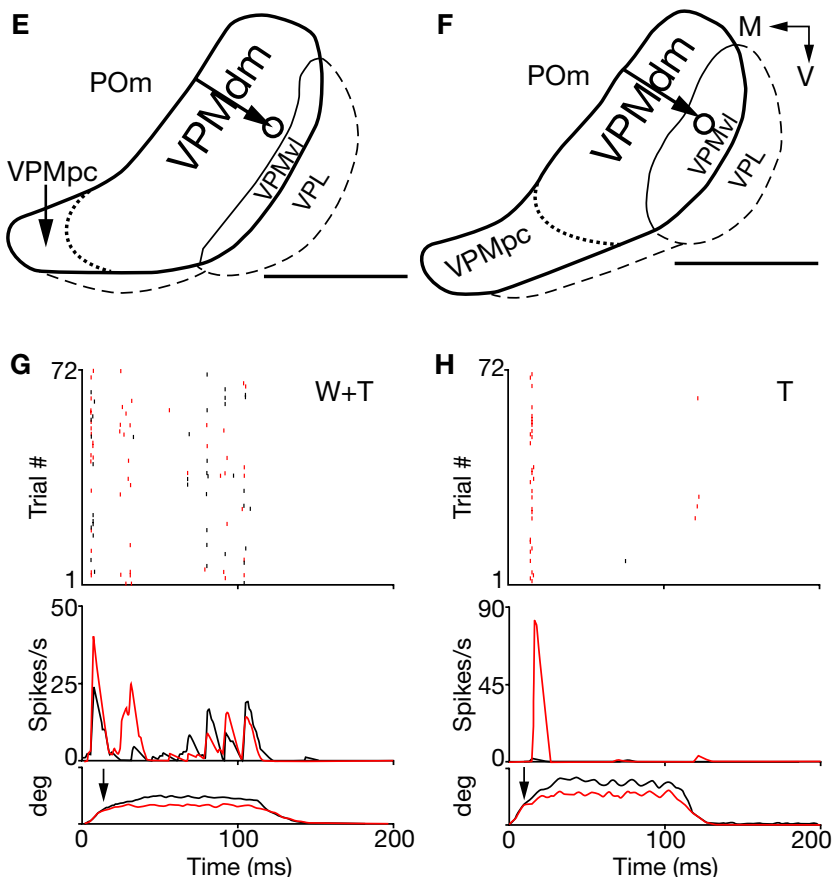

Figure 6 | Morphological and functional properties of the VPMdm and VPMvl along their rostrocaudal extent. $(\boldsymbol{A}, \boldsymbol{B})$. Coronal slices through the VPM at about -3.0 and $-3.7 \mathrm{~mm}$ caudal to bregma (9th and 16th of 24 consecutive slices), respectively. Arrows indicate recording site lesions. (C,D) Canonical views of oblique slices through the VPM at the level of arcs 3-4. Vertical lines correspond to coronal slices in $(\boldsymbol{A}, \boldsymbol{B})$, respectively. Lesion locations are marked by circles. $(\boldsymbol{E}, \boldsymbol{F})$ Contours of the VPMdm and VPMvl in the coronal slices of the thalamus depicted in $(\boldsymbol{A}, \boldsymbol{B})$, respectively. Dotted lines separate the VPMpc from the rest of the VPM. Lesion locations are indicated by arrows. $(\boldsymbol{G}, \boldsymbol{H})$ Responses of individual neurons from the recording sites in $(\boldsymbol{A}, \boldsymbol{B})$, during steady-state for the VPMdm (G) and VPMvl $(\boldsymbol{H})$, respectively, to whisking in air (black) and against an object (red), relative to the time of protraction onset $(t=0)$. For both neurons, a raster display of single spikes accumulated from three randomly selected cycles from cycles 5-10 is depicted by the top graph. Peristimulus time histograms (PSTHs) computed for the entire steady-state period (cycles 5-10) are depicted in the middle. The horizontal angle of the principal whisker during a single cycle is depicted at the bottom; the ordinate denotes whisker angle, with full scale $=50^{\circ}$; traces were low pass filtered at $160 \mathrm{~Hz}$. Contact times during touch trials are indicated by arrows. DM, dorsomedial; M, medial; R, rostral; T, touch signals; V, ventral $\mathrm{W}+\mathrm{T}$, additive whisking and touch signals. Scale bars are $1 \mathrm{~mm}$. thalamus. Construction of the $\mathrm{dm} / \mathrm{vl}$ border in the VPM was derived from non-standard oblique slices, in which the border was identified anatomically, using a normalized linear coordinate transformation.

In oblique, dorsomedial to ventrolateral slices of young rat VPM, septa that separate the core from the tails of macrobarreloids were revealed for the first time. These septa exhibit weak $\mathrm{CO}$ activity, and contain sparse cell bodies (see Figure 4). Thus, the two compartments ( $\mathrm{dm}$, which contains cores of barreloids, and vl, which contains the tails) of the VPM can be easily differentiated in young rats. As rats mature, the septa in their VPM become less clear. In adult rats, the septa between the cores and tails of barreloids are narrow, and contain more axonal bundles than neighboring barreloid cores or tails. The septa contain myelinated fibers that fulfill a conductive function. Other functions of septa in the VPM were discussed by Pierret et al. (2000) who specified the region between the VPMdm and VPMvl as a transition zone that contains cells that may receive convergent inputs from both PrV and SpVi afferents. These authors concluded that the transition zone was "ill-defined" with regard to both the topographic distribution of trigeminal inputs and the dendroarchitecture of the relay cells. In oblique slices that revealed both barreloid arcs (see Figures 3A and 4) and entire barreloid fields (Figures 7A-F), septa between individual barreloids along the arcs, as well as along the rows, were clearly seen in both young and adult rats. Interbarreloid septa were first described in the VPM of mice by Van der Loos (1976), who occasionally observed a septum of low cell density similar to, but thinner than, the septa that separate the large cortical barrels. In the VB of neonatal rats, discrete curvilinear arrays of cells that are separated from each other by narrow, cell-sparse zones were described by Ivy and Killackey (1982), using retrograde transport of horseradish peroxidase. In rat VPM stained for CO activity, a series of segregated oval patches of intense CO activity that are separated from each other by narrow zones in which $\mathrm{CO}$ activity is considerably lower was observed by Land and Simons (1985). Land et al. (1995) concluded that the distinctive appearance of barreloids in oblique planes is partly caused by the arrangement of fiber bundles entering and leaving the nucleus via the trigeminal lemniscus and internal capsule, and passing between individual rows and arcs. The functional significance of interbarreloid septa may be related to the fact that they contain neuronal projections from the SpVi. These projections were revealed by rhodamine dextran injections into the SpVi (Williams et al., 1994).

In the present study, staining for $\mathrm{CO}$ activity revealed different patterns of neuronal organization within the VPM. The ventromedial part of the VPM, which contains small neurons and is known as the parvicellular part of the VPM (VPMpc), appeared as a homogenously and strongly stained sub-structure in the coronal and both oblique planes used (see Figures $3 \mathrm{~A}$ and 7E,F). The VPMpc extended from $\sim 0.3 \mathrm{~mm}$ to $\sim 1.5 \mathrm{~mm}$ laterally from the midline. Detailed analysis of consecutive oblique slices of the rest of the VPM revealed a highly organized, dorsomedial compartment of the VPM (VPMdm) that contained the cores of barreloids, and a ventrolateral compartment (VPMvl) that contained the tails of barreloids. In oblique slices, in the dorsolateral to ventromedial direction, the entire VPMdm (along the "long axis" of the VPM) can be divided into two specifically organized parts: (1) a dorsolateral part (up to $1 \mathrm{~mm}$ in length), which contains macrobarreloids with transversal diameters of $\sim 0.1 \mathrm{~mm}$ along rows, and $\sim 0.2 \mathrm{~mm}$ along arcs, and which can be referred to as the "macrovibrissal part"; and (2) a more ventromedial 

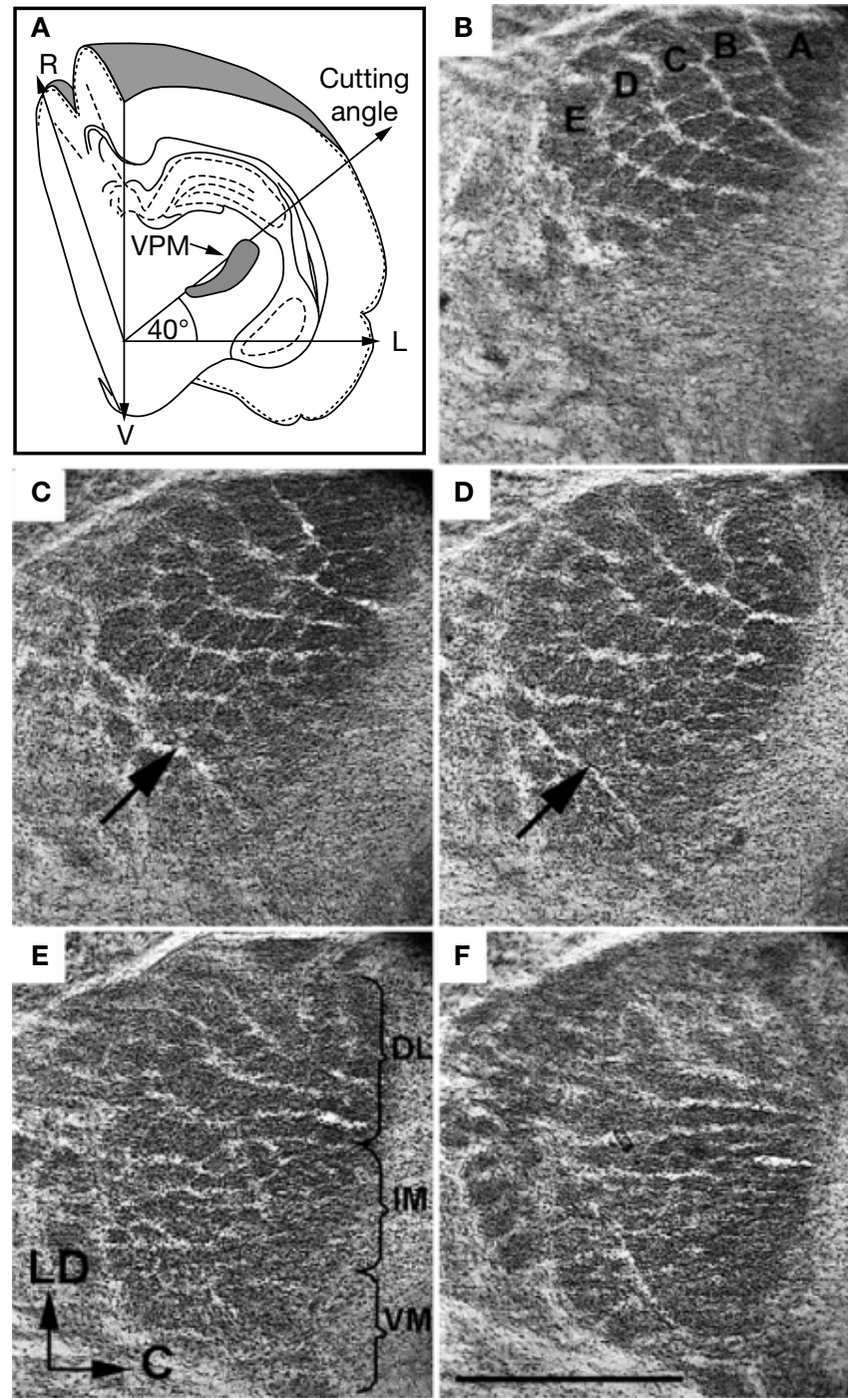

Figure 7 | Visualization of an entire barreloid field in an oblique $\left(40^{\circ}\right.$ to the horizontal plane, from dorsolateral to ventromedial) plane of a 9-day-old rat. (A) Schematic drawing demonstrating the cutting plane. (B-F) Alternate oblique, $70 \mu \mathrm{m}$ thick, slices through the VPM. Arrows in $(\boldsymbol{C}, \boldsymbol{D})$ indicate the septum that separates the rostral part of the intermediate portion of the VPM from the caudal part. A, B, C, D, and E indicate barreloid rows. DL, dorsolateral; IM, intermediate; VM, ventromedial portions of the VPM; L, lateral; LD, laterodorsal; $R$, rostral; $V$, ventral. Scale bar is $1 \mathrm{~mm}$.

part, $1.2-1.5 \mathrm{~mm}$ in length, that extends between the macrovibrissal part and VPMpc, and which contains many thin barreloids (with diameters $\leq 0.1 \mathrm{~mm}$ ), and which can be referred to as the "intermediate part." The highly reproducible appearance of VPM compartments facilitated our proposal of a model of the compartmentalization of the rat VPM (Figure 8). Each compartment is illustrated with typical oblique sections (slices) that contain macrobarreloids ( $\mathrm{a}$ and $\mathrm{b}$ ), thin barreloids (c), or a homogenously-stained substrate (d). Structural peculiarities of the vibrissal and intermediate parts of the VPM were not usually visible in coronal slices, but were easily seen in oblique slices.

In this and our previous (Haidarliu and Ahissar, 2001) study, barreloids appear as rod-like structures, with almost constant values $(\sim 100 \times 200) \mu \mathrm{m}$ for their two short axes, while that of the third is represented by a length gradient. The VPMdm, which contains the cores of barreloids, displays the most $\mathrm{CO}$ activity

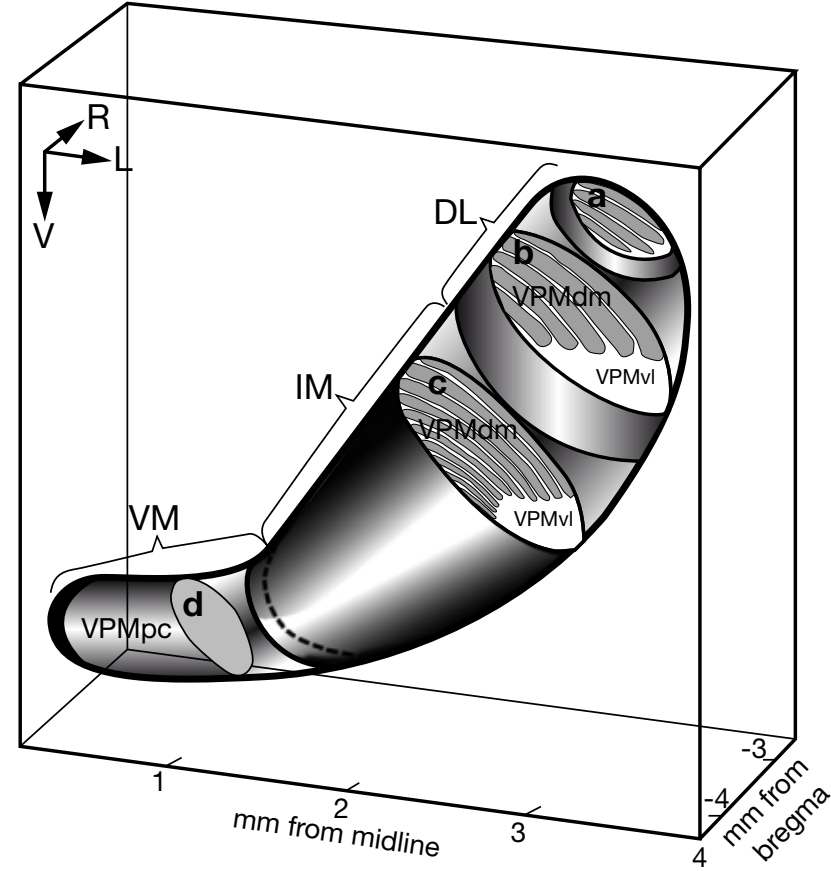

Figure 8 | Three-dimensional model of the intrinsic organization of the rat VPM. DL, the dorsolateral portion, which contains large barreloids that represent four straddlers (section a) and five rows of mystacial vibrissae (section b). IM, the intermediate portion, which contains multiple thin barreloids (section c). VM, ventromedial portion, which is known as the parvicellular compartment of the VPM (VPMpc), and which stains uniformly (section d). The border of the VPM is depicted by a thick solid line. The thick dotted line separates the IM portion from the VM. a, b, c, and d are schematic oblique cutting planes through the VPM. L, lateral; $R$, rostral; $V$, ventral.

of the VPM. In young rats, tissue components that stained for $\mathrm{CO}$ activity, especially cell bodies, were evenly distributed within each of the barreloids in the core compartment. In adult rats, many unstained strips of axonal bundles passed through the core of barreloids and the septa. The ventrolateral (vl) and caudal parts of the VPM, which contain multiple axonal bundles, and are not considered to display a typical barreloid arrangement, exhibit less CO activity. However, the VPMvl does contain barreloid continuations ("tails"), which are curved along their long axes and are visualized when cut at specific angles or anterogradely labeled with biotinylated dextran amine injected into the SpVi (Pierret et al., 2000). The shape of the VPMvl revealed by our measurements is consistent with the observations of Pierret et al. (2000) that when SpVi terminal fields are visualized in coronal sections of the VPM with biotinylated dextran amine, the VPMvl is thicker caudally, and narrows rostrolaterally (see also Figures 3 and 5). Injection of biotinylated dextran amine in the infragranular layers of the rat S2 reveal corticothalamic terminals in consecutive coronal slices (Bokor et al., 2008), the distribution of which appears to be within the boundaries of the VPMvl determined in the present study.

Of the three VPM parts (dorsolateral, intermediate, and ventromedial), the intermediate one seems to be the most complicated structurally and functionally. The dorsolateral portion of the intermediate part contains thin barreloids, which represent microvibrissae and hairy buccal pads. More ventromedially, barreloids are observed only in the rostral part of the intermediate portion (Figure $3 \mathrm{~A}$, slice 22 ), which is separated from the 
caudal part by a septum (Figures 7C,D, arrows) that does not stain for CO activity. Such segregation may be related to the spatial separation of regions relaying different sensory modalities. Data about the exact location of these regions varies, depending on the sensory modality studied. For example, representations of periodontal mechanosensitive neurons occurred in the most ventral part of the intermediate portion of the VPM (Tabata et al., 2002), while oral nociceptive sensations were relayed mostly by the rostral half of the VPM (Park et al., 2006; Zhang et al., 2006). So the intermediate segment of the VPM differs from the other two VPM segments by relaying sensory pathways of different modalities.

In summary, mapping of the structural organization of the rat VPM was performed by cutting rat brains at different angles, staining brain slices for CO activity, and translating anatomical coordinates from one brain cutting plane to another. Extensive staining for $\mathrm{CO}$ activity was used to microscopically define individual vibrissal representations and as to establish intrinsic organization of the VPM sub-nuclei. The present study revealed that the dorsolateral to ventromedial extent of the VPM consists of three specifically organized parts: (1) a macrovibrissal part that is composed of large barreloids that represent macrovibrissae of the mystacial pad; (2) an intermediate part that contains large number of thin barreloids that relay small vibrissae as well as head representations of different sensory modalities; and (3) a parvicellular part that is adjacent to the intermediate part of the VPM, and relays gustatory representations. In the vibrissal part of the VPM, we defined the border between the two sub-nuclei, the VPMdm and VPMvl, which was mapped onto images of coronal slices along the rostrocaudal extent of the VPM. Mapping of these sub-nuclei can augment rat brain atlases, facilitate target localization in relevant parts of the VPM, and enable association of neuronal function with its corresponding afferent pathway.

\section{CONFLICT OF INTEREST STATEMENT}

The authors declare that the research was conducted in the absence of any commercial or financial relationships that could be construed as a potential conflict of interest.

\section{ACKNOWLEDGEMENTS}

This work was supported by The United States-Israel Binational Science Foundation, European Commision grant BIOTACT (ICT-215910), The Minerva Foundation funded by the Federal German Ministry for Education and Research, and Israel Science Foundation. Ehud Ahissar holds the Helen Diller Family Professorial Chair of Neurobiology. We thank Dr Barbara Schick for reviewing the manuscript.

\section{REFERENCES}

Belford, G. R., and Killackey, H. P. (1979). Vibrissae representation in subcortical trigeminal centers of the neonatal rat. J. Comp. Neurol. 183, 305-322.

Bokor, H., Acsady, L., and Deschenes, M. (2008). Vibrissal responses of thalamic cells that project to the septal columns of the barrel cortex and to the second somatosensory area. J. Neurosci. 28, 5169-5177.

Cechetto, D. F., and Saper, C. B. (1987). Evidence for a viscerotopic sensory representation in the cortex and thalamus in the rat. J. Comp. Neurol. 262, 27-45.

Davidson, N. (1965). The projection of afferent pathways on the thalamus of the rat. J. Comp. Neurol. 124, 377-390.
Emmers, R. (1965). Organization of the first and the second somesthetic regions (SI and SII) in the rat thalamus. J. Comp. Neurol. 124, 215-228.

Groenewegen, H. J., and Witter, M. P. (2004). Thalamus. In The Rat Nervous System, G. Paxinos, ed. (Amsterdam, Elsevier), pp. 407-453.

Haidarliu, S., and Ahissar, E. (2001). Size gradients of barreloids in the rat thalamus. J. Comp. Neurol. 429, 372-387. Erratum in: J. Comp. Neurol. 431, 127-128.

Haidarliu, S., Sosnik, R., and Ahissar, E. (1999). Simultaneous multi-site recordings and iontophoretic drug and dye applications along the trigeminal system of anesthetized rats. J. Neurosci. Methods 94, 27-40.

Ivy, G. O., and Killackey, H. P. (1982). Ephemeral cellular segmentation in the thalamus of the neonatal rat. Dev. Brain Res. 2, 1-17.

Kosar, E., Grill, H. J., and Norgren, R. (1986). Gustatory cortex in the rat. II. Thalamocortical projections. Brain Res. 379, 342-352.

Land, P. W., and Akhtar, N. D. (1987). Chronic sensory deprivation affects cytochrome oxidase staining and glutamic acid decarboxylase immunoreactivity in adult rat ventrobasal thalamus. Brain Res. 425, 178-181.

Land, P. W., Buffer, S. A. Jr, and Yaskosky, J. D. (1995). Barreloids in adult rat thalamus: three-dimensional architecture and relationship to somatosensory cortical barrels. J. Comp. Neurol. 355, 573-588.

Land, P. W., and Simons, D. J. (1985). Metabolic and structural correlate of the vibrissae representation in the thalamus of the adult rat. Neurosci. Lett. 60, 319-324.

Macchi, G. (1983). Old and new anatomo-functional criteria in the subdivision of the thalamic nuclei. In Somatosensory Integration in the Thalamus, G. Macchi, A. Bustioni and R. Spreafico, eds (Amsterdam, Elsevier), pp. 3-15.

Park, S. J., Zhang, S., Chiang, C. Y., Hu, J. W., Dostrovsky, J. O., and Sessle, B. J. (2006). Central sensitization induced in thalamic nociceptive neurons by tooth pulp stimulation is dependent on the functional integrity of trigeminal brainstem subnucleus caudalis but not subnucleus oralis. Brain Res. 1112 134-145.

Paxinos, G., and Watson, C. (1998). The Rat Brain in Stereotaxic Coordinates, 4th edn. New York, Academic.

Pierret, T., Lavallee, P., and Deschenes, M. (2000). Parallel streams for the relay of vibrissal information through thalamic barreloids. J. Neurosci. 20, $7455-7462$

Price, J. L. (1995). Thalamus. In The Rat Nervous System, G. Paxinos, ed. (San Diego, Academic), pp. 629-648.

Saper, C. B. (1995). Central autonomic system. In The Rat Nervous System, G. Paxinos, ed. (San Diego, Academic) pp. 107-135.

Sugitani, M., Yano, J., Sugai, T., and Ooyama, H. (1990). Somatotopic organization and columnar structure of vibrissae representation in the rat ventrobasal complex. Exp. Brain Res. 81, 346-352.

Swanson, L.W. (1992). Brain Maps: Structure of the Rat Brain. New York, Elsevier.

Szwed, M., Bagdasarian, K., and Ahissar, E. (2003). Encoding of vibrissal active touch. Neuron 40, 621-630.

Tabata, T., Yamaki, A., Takahashi, Y., and Hayashi, H. (2002). Physiological properties of periodontal mechanoreceptive neurons in the posteromedial ventral nucleus of rat thalamus. Arch. Oral Biol. 47, 689-694.

Urbain, N., and Deschenes, M. (2007). A new pathway of vibrissal information modulated by the motor cortex. J. Neurosci. 27, 12407-12412.

Vahle-Hinz, C., and Gottschaldt, K.-M. (1983). Principal differences in the organization of the thalamic face representation in rodents and felids. In Somatosensory Integration in the Thalamus, G. A. Macchi, A. Bustioni and R. Spreafico, eds (Amsterdam, Elsevier), pp. 125-145.

Van der Loos, H. (1976). Barreloids in mouse somatosensory thalamus. Neurosci. Lett. 2, 1-6.

Veinante, P., and Deschenes, M. (1999). Single- and multi-whisker channels in the ascending projections from the principal trigeminal nucleus in the rat. J. Neurosci. 19, 5085-5095.

Verhagen, J. V., Giza, B. K., and Scott, T. R. (2003). Responses to taste stimulation in the ventroposteromedial nucleus of the thalamus in rats. J. Neurophysiol. $89,265-275$.

Waite, P. M. E. (1973). Somatotopic organization of vibrissal responses in the ventro-basal complex of the rat thalamus. J. Physiol. (Lond.) 228, 527-540.

Williams, M. N., Zahm, D. S., and Jacquin, M. F. (1994). Differential foci and synaptic organization of the principal and spinal trigeminal projections to the thalamus in the rat. Eur. J. Neurosci. 6, 429-453.

Yu, C., Derdikman, D., Haidarliu, S., and Ahissar, E. (2006). Parallel thalamic pathways for whisking and touch signals in the rat. PLoS Biol. 4, e124.

Zhang, S., Chiang, C. Y., Xie, Y. F., Park, S. J., Lu, Y., Hu, J. W., Dostrowsky, J. O., and Sessle, B. J. (2006). Central sensitization in thalamic nociceptive neurons induced by mustard oil application to rat molar tooth pulp. Neuroscience 142, 833-842. 